\title{
Letters to the
}

\section{Editor}

\section{Carpentier-Edwards Perimount valve and intraoperative structural failure To the Editor:}

We read with great interest the article by Saunders and associates. ${ }^{1}$ The authors described a post-cardiopulmonary bypass (post-CPB) structural valve failure, leading to an intraoperative replacement of 4 Carpentier-Edwards Perimount valves. The negative experience has been lived in 2 different institutions. Two Perimount valves were replaced because of a severe central insufficiency at the weaning-off phase from $\mathrm{CPB}$, another valve was replaced soon after its insertion because of being judged not continent as a result of the impossibility of leaflet coaptation before atrial closure, and the last as a result of incompetence at hydraulic testing. The pericardial tissue valves were replaced with either a new Carpentier-Edwards porcine valve or a Medtronic Mosaic porcine valve. The authors speculated that the Perimount valves were distorted by the mitral valve annulus, leading to an unacceptable incompetence, and that the tolerance to this mechanical distortion is lower for pericardial tissue valves when compared with porcine valves.

The Carpentier-Edwards tissue valves, both porcine and pericardial, have the same support. ${ }^{2}$ The stent is an Elgiloy wire support, which allows a specific area flexibility, namely at the post area, with the aim to reduce the leaflet stress at the commissures. The bovine pericardium is glutaraldehyde fixed at zero pressure to enhance tissue durability. One of the limits related to the no-pressure fixation method is the reduced leaflet coaptation when the valve is packaged. Nevertheless, when the valve is exposed to normal physiologic pressure, namely left ventricular pressure during systole or diastolic aortic pressure according to the insertion position, the leaflets coapt, and the valves function well.

The first valve has been replaced before left atrial closure. Therefore its perfor- mance was not physiologically tested. The second Carpentier-Edwards Perimount valve has been replaced after a long period of CPB. In the second patient the Perimount valve showed incompetence after hydraulic testing, and therefore it has been removed. The last patient experienced massive native mitral and tricuspid valve incompetence, and he was referred with a normal left ventricular ejection fraction. Immediately after weaning from $\mathrm{CPB}$, the valve showed severe central incompetence at transesophageal echocardiography, and this valve was replaced.

Our hypothesis is that in all these cases the tissue valves were not exposed to enough physiologic pressure to allow normal leaflet coaptation, provoking a misleading incompetence that convinced the surgeons to replace the valves and to retain the cause of failure related to a structural failure. The early primary structural failure of a tissue valve is a well-defined entity, and it is believed to occur when part of the elements of a prosthesis are not well functioning because of wear and tear. We think that if these replaced prostheses were tested at physiologic pressure in vitro or in vivo, they would show a normal function.

Tomaso Bottio, MD, PhD Vincenzo Tarzia, MD

Claudio Muneretto, MD

Department of Cardiovascular Surgery University of Brescia Medical School Brescia, Italy

\section{References}

1. Saunders PC, Grossi EA, Esposito RA, Bizekis CS, Strong MD, Colvin SB. Failure of four bovine pericardial mitral prostheses. J Thorac Cardiovasc Surg. 2004;127:267-8.

2. Jamieson WRE, Marchand MA, Pelletier CR, Norton R, Pellerin M, Dubiel TW, et al. Structural valve deterioration in mitral replacement surgery: comparison of Carpentier-Edwards supra-annular porcine and Perimount pericardial. $J$ Thorac Cardiovasc Surg. 1999;118:297-305.

doi:10.1016/j.jtcvs.2004.04.045 